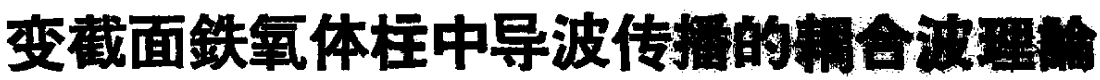

\author{
黄宏嘉范㣀元
}

微波技术中经常应用盖法拉第旋转器件。它的 最普通形式是一段圆柱形波导，其中放有一根䋱向 磁化的铁載体柱; 为了減小反射，铁氧体杜两端制 成截面渐变的雉形。据作者所知，对这种变截面的 铁氧体柱，文献中还未曾发表过任何完整的理论分 析文点。在本文中，作者尝试用耦合波理论来处理 这个复杂的电磁波传播问题。

利用正交函数展开的方法首先导出了一组适用

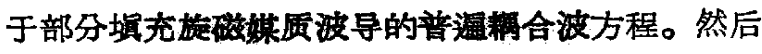
研究了在始向磁化的具有变截面对湤段的铁氧体柱 中导波的法拉第族转。考虑 $H_{11}$ 偶波和奇波，利用 缓变系数法 [数学学报, 11, 238(1961)] 导出了下 列的普㵓会式:

$$
\theta=\frac{1}{u_{11}^{2}-1} \frac{1}{j_{1}^{2}\left(u_{11}\right)} \frac{\kappa}{\mu_{0}} \beta_{11} \int_{0}^{l} j_{1}^{2}\left(u_{11} \frac{b}{a}\right) d z
$$

式中， $\theta$ 是法拉第旋转角; $a$ 是圆波导牛径; $b$ 是 铁氧体柱牛径 (随 $z$ 改变)； $l$ 是安放在轴线方向 （ $z$ 方向）的变截面铁氧体柱的全长; $J_{1}$ 是一阶贝塞 尔函数， $u_{11}$ 是 $J_{1}^{\prime}=0$ 的第一个非零根； $\beta_{11}$ 是 $H_{11}$ 波的未微扰相位常数; $\mu_{0}$ 是冥室导 磁 系 数; $\kappa$ 是 铁等体材料橫向导磁系数扑矢中任一对角线外元的 数值。

对于对称结构，从上列普逃公式可以导出下列 近似计算公式:

$$
\begin{aligned}
\theta= & 0.349 \frac{\kappa}{\mu_{0}} \beta_{11}\left(\frac{b_{0}}{a}\right)^{2}\left(3 l-4 l_{1}\right)- \\
& -0.177 \frac{\kappa}{\mu_{0}} \beta_{11}\left(\frac{b_{0}}{a}\right)^{4}\left(5 l-8 l_{1}\right)
\end{aligned}
$$

式中 $l_{1}$ 是两头任一变载面过渡段的长度。

过去文献中其他作者利用不同方法得到的各种 法拉第旋转角公式都可以从上列的普遍公式导出，
因而可潘成是这个普造公式的特例。则如，对均与 截面铁呠体柱来说，普遥公式化为

$$
\theta=\frac{1}{u_{11}^{2}-1} \frac{\kappa}{\mu_{0}} \beta_{11}\left[\frac{J_{1}\left(u_{11} b_{0} / a\right)}{J_{1}\left(u_{11}\right)}\right]^{2} \iota
$$

这正是 Severin 利用数扰法导出过的公式(见 Trans. IRE, MTT-7, 337 页)。

另一方面，对完全填充铁奥体波导来说，普㴜 公式简化为

$$
\theta=\frac{1}{u_{11}^{2}-1} \frac{\kappa}{\mu_{0}} \beta_{11} l
$$

这个公式和 Suhl 与 Walker 导出的相同 (BSTJ, 33 急, 1133 卉)。

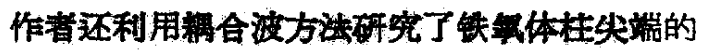
反射。在反射问题中，考虑了四个波的裸合问题。 这四个波是前向的 $H_{11}$ 偶波和奇波以及 后向的 $H_{11}$ 偶波和薮波。利用缓变系数法扭尔取若千近似，导 出了下列的辰射系数公式:

$$
\begin{gathered}
R=\int_{0}^{l} e^{-i f \int_{0}^{*} \beta^{+} d x^{\prime}}\left[C^{+} \sin \left(\int_{0}^{x} 2 C^{+} d z^{\prime}\right)-\right. \\
\left.-j C^{-} \cos \left(\int_{0}^{z} 2 C^{+} d z^{\prime}\right)\right] d x
\end{gathered}
$$

式中， $\beta^{+}$基前间 $H_{11}$ 偶波或奇波的撒扰 后橧 位 常 数； $C^{-}$是前后向 $H_{11}$ 偶波之间或奇波之间的耦合采 数； $C^{+}$是 $H_{11}$ 偶波和奇波之间的耦合系数。

在特殊的情形下，如果铁等体杜的截面是均与 的，反射系数的公式化为

$$
\begin{aligned}
R_{0}= & \frac{e^{-2 j \beta_{0}^{+} l}}{2\left(\beta_{0}^{+2}-C_{0}^{+2}\right)}\left\{C _ { 0 } ^ { + } \left(j \beta_{0}^{+} \sin 2 C_{0}^{+} l+\right.\right. \\
& \left.+C_{0}^{+} \cos 2 C_{0}^{+} l\right)-j C_{0}^{-}\left(j \beta_{0}^{+} \cos 2 C_{0}^{+} l-\right. \\
& \left.\left.-C_{0}^{+} \sin 2 C_{0}^{+} l\right)\right\}-\frac{C_{0}^{+2}+C_{0}^{-} \beta_{0}^{+}}{2\left(\beta_{0}^{+2}-C_{0}^{+2}\right)}
\end{aligned}
$$


式中，符号的下标““像”是指在 $b(z) \equiv b_{0}$ 处的值。

利用耦合波方法还研究了铁氧体柱的几何小变 形问题。

为此, 将铁氧体柱牛径表示为

$$
b(\varphi)=b_{0}\left[1+\sum_{p=1} \delta_{p} \cos p\left(\varphi-\psi_{p}\right)\right]
$$

$b_{0}$ 是平均牛径, $\psi_{p}$ 规定了变形铁氧体柱的 取 向; $p=1$ 描写了偏离轴线的情形, $p=2$ 描写了椭圆 度，等等。

根据耦合波理论，任何形式的几何变形都会在 有关的耦合系数中反映出来。导出了耦合系数, 就 不难计算在有变形情形下的法拉第旋转角:

$\theta \approx C_{0}^{+}+l\left\{1+\frac{\delta_{1}^{2}+\delta_{2}^{2}}{2}\left(u_{11} \frac{b_{0}}{a}\right)^{2}\left[\left(\frac{J_{1}^{\prime}}{J_{1}}\right)^{2}+\frac{J_{1}^{\prime \prime}}{J_{1}}\right]+\right.$

$$
\begin{aligned}
& +\frac{\left(\delta_{2} \sin 2 \varphi_{2}\right)^{2}}{8\left(\frac{\kappa}{\mu_{0}}\right)^{2}}\left[\frac{\omega^{2} \mu_{0}\left(\varepsilon-\varepsilon_{0}\right)}{\beta_{11}^{2}}+\right. \\
& \left.\left.+\frac{\mu-\mu_{0}}{\mu_{0}}\right]^{2}\left[1-\left(u_{11} \frac{b_{0}}{a}\right)^{2}\left(\frac{J_{1}^{\prime}}{J_{1}}\right)^{2}\right]\right\}
\end{aligned}
$$

式中贝塞尔函数的宗量是 $u_{11} b_{0} / a_{\text {。 }}$

这个公式解释了实验中发现的一个现象, 即椭 圆度对法拉第旋转的影响决定于铁佱体柱的取向; 当 $\varphi_{2}=0$ 或 $\pi / 2$ 时（入射 $H_{11}$ 波的电場方向和椭圆 的长轴或短轴重合), 影响最小; 当 $\varphi_{2}=\pi / 4$ 时(电 場方向介于长短轴方向之间), 影响最大。

塊合波理论对所硏究的具有非均匀几何的铁氧 体问题特別有用。这类问题如果利用以往研究微波 铁氧体的标准分析方法 (如微扰法、变分法等)来处 理是很难求得结果的。

\title{
三价希土氧化物一一氧化鈹系統的研究
}

\section{III. $\mathrm{Gd}_{2} \mathrm{O}_{3}$ - $\mathrm{BeO}$ 系統的平衡与介稳平衡关系}

\author{
韓文龙郭稆崖
}

（中国科学院硅酸盐化学与工学研究所）

在 $\mathrm{La}_{2} \mathrm{O}_{3}-\mathrm{BeO}$ 二元系中有 $\mathrm{La}_{2} \mathrm{O}_{3} \cdot 2 \mathrm{BeO}$ 和 $3 \mathrm{La}_{2} \mathrm{O}_{3} \cdot 2 \mathrm{BeO}$ 两个化合物 ${ }^{[1]}$ ，其他三价希土氧化物 一一氧化鈹的化合物则掎未见诸文献报导。

Тресвятский 等 ${ }^{[2]}$ 由高溫热分析法作出的 $\mathrm{Gd}_{2} \mathrm{O}_{3}-\mathrm{BeO}$ 系统的相图为简单共熔二元系，未发 现有任何形式的中间化合物。然而在他们作出的相 图上，共熔点已低于氧化鈹熔点 $1000^{\circ} \mathrm{C}$ 以上，可设 想熔体中有铍氧复合离子生成 ${ }^{[3]}$, 如果某种鈹氧复 合离子能维持至发生结晶作用以后佮有一定的稳定 区域, 则将出现新化合物。

因此，为了探讨三价希土氧化物与氧化鈹之间 生成的化合物, 对于 $\mathrm{Gd}_{2} \mathrm{O}_{3}-\mathrm{BeO}$ 系统的相 平衡关 系重新作了研究。
由淬火法 ${ }^{[1]}$ 与结合淬冾后试样的偏光显微镜和 $\mathrm{X}$-射线粉末法分析的结果，作出 $\mathrm{Gd}_{2} \mathrm{O}_{3}-\mathrm{BeO}$ 系统 的相图，绘于图 1。

我们测得的相图与 Тресвятский 等的结果相似， 为简单共莶二元系, 只是富舞化鲏部分的液相线较 Тресвятскии 等测定者为高, 最大相差约 $100^{\circ} \mathrm{C}$ 。由 淬火法更精确地测得的共熔点为: $1472 \pm 2^{\circ} \mathrm{C} 、 53.4$

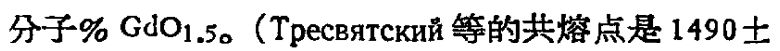
$\pm 10^{\circ} \mathrm{C} 、 35$ 分子 $\% \mathrm{Gd}_{2} \mathrm{O}_{3}$, 郎 51.9 分子 $\% \mathrm{GdO}_{1.5}$ )

此外，本工作中还发现了一种新结晶相。其折 光指数 $n_{p}=1.89 、 n_{g}=1.94$, 为二轴晶, 光轴角 接近于 $90^{\circ}$ 。图 2 列出其 $X$ - 射线粉末法 照相 及与 氧化鈹和氯化釷照相的比较,根据新相的衍射谱线， 